\title{
High-Stroke Motion Modelling and Voltage/Frequency Proportional Control of a Stick-Slip Microsystem
}

\author{
Micky Rakotondrabe, Yassine Haddab and Philippe Lutz
}

\begin{abstract}
A new control type for stick-slip microsystems is proposed in this paper: the voltage/frequency (U/f) proportional control. It gives a best resolution relatively to the classical control algorithm. It is also an englobalization of three classical controllers: the sign controller, the classical proportional controller and the frequency proportional controller. A high stroke model of a stick-slip microsystem is first given. Then, we theoretically analyze the performances of the closed loop process with the $U / f$ controller. Finally, we give some experimental results obtained with different values of the proportional gains.
\end{abstract}

\section{INTRODUCTION}

Among the stepping principles for micropositioning, stick-slip [1] is the most used : examples are given in [2][3][4][5]. Stick-slip actuators, generally based on piezoelectric materials, are characterized by their simplicity, rapidity, low cost and possibility of batch fabrication. Two modes of motion can be obtained with a stickslip micropositioner [6] : the stepping mode and the scanning mode. The stepping mode consists in applying a sawtooth voltages to the micropositioner and let it move step by step, in high range and with a high velocity on the workspace (Fig. 1-a, b and c). The resolution in this mode is limited to one step. When the difference between the target position and the current position becomes less than the value of a step, the legs (piezoelectric actuators) are bent slowly until the final position is reached (Fig. 1d). This is the scanning mode and the obtained resolution can be very high. It is possible to have a similar resolution in the stepping mode by reducing the amplitude $U$ of the sawtooth but the vibrations occuring in each steps [7] may influence the performances.

Two modes of control for stick-slip micropositioners are possible: control in stepping mode for high stroke positioning and control in scanning mode for fine positioning. Each of them may use an open-loop or a closedloop structure. As the fine positioning may be controlled in closed loop with standard controllers (PID, optimal controllers, robust controllers, etc.), this paper deals with the high stroke positioning control.

The first section is a brief presentation of existing control methods. Then, we present the high stroke characteristics and model our stick-slip microsystem for ex-

Laboratoire d'Automatique de Besançon

- CNRS UMR6596, ENSMM - Université de Franche-Comté,

25000 Besançon - France

\{mrakoton, yhaddab, plutz\}@ens $2 m$.fr periments. After that, we detail a new type of control : the voltage/frequency proportional control. It inferes good resolution of the motion relative to the existing methods. On the other hand, the vibrations possibilities are reduced in this approach. Finally, we present the experimental and simulation results.

(a)
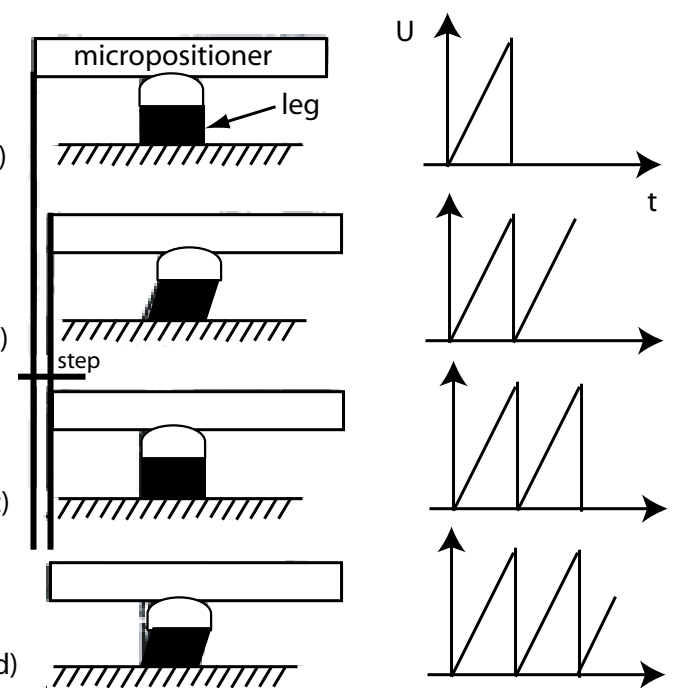

Fig. 1. Stick-slip principle [6]. a, b and c : stepping mode. d : scanning mode.

\section{EXISTING METHODS}

Open-loop control based on step counting as in stepper motors are not applicable for stick-slip microsystems because:

- the steps are never identical along a displacement due to the nonlinearities phenomena coming from the friction and the piezoelectric materials,

- the step is very low relative to the distance, then the number of steps of the displacement is very high. Thus, small counting error may generate high static error,

- the step errors $\left(s t e p_{\text {errors }}=s t e p_{\text {estimated }}-s_{\text {step }} p_{\text {real }}\right)$ accumulate along the motion unlike those of in stepper motors,

- the external disturbances (adhesion forces, thermal effect, vibrations of the workspace etc.) influence 
the accuracy.

Thus, the classical employed closed-loop controller is the following algorithm [8] except for a sign:

$$
\begin{aligned}
& \text { While }\left|x_{c}-x\right| \geq \text { step Do } \\
& \quad \text { apply one step } \\
& \text { EndWhile }
\end{aligned}
$$

where $x_{c}$ is the target position and $x$ is the current position. When the accuracy of the sensor is worse than the value of a step, the term step in the precedent algorithm is replaced by $n \times$ step which gives the limit of the sensor accuracy.

Another method based on the hybrid modeling of the stick-slip systems has been proposed in [9]. The hybrid model is approximated into a continuous one (called dehybridization) and a continuous controller is applyed $(P D)$. This method gives the possibility to reach the target position without using two separate control modes (stepping and scanning). However, the dehybridization requires a hybrid controller in series with the stickslip microsystem which makes the whole algorithm more complex than the precedent algorithms.

Finally, Breguet and Clavel proposed a numerical frequency proportional controller in [10]. In this method, the frequency is proportional to the error while the amplitude $U$ of the voltage still stays constant. Thereby, as the step is constant along the motion, the resolution is also constant.

\section{Characteristics And MODElling of The MICROSYSTEM}

Fig. 2-a shows the stick-slip microsystem [11] used for experiments in this paper. Fig. 2-b shows how the microactuators are spread out inside the microsystem. It has two degrees of freedom (2DoF: linear and angular) but our test will only be performed in the linear motion. The maximal step value of the microsystem is about $200 \mathrm{~nm}$ and the speed can reach $2 \mathrm{~mm} / \mathrm{s}$. These performances are obtained using a sawtooth input voltage with an amplitude of $\pm 150 \mathrm{~V}$ and a frequency of $10 \mathrm{kHz}$.

When applying a continuous input voltage $U$, the microsystem works in scanning mode. Hypothesizing that the scanning displacement $x_{s c}$ is dynamically linear reative to the voltage, we can write the following function:

$$
X_{s c}(s)=G_{s c}(s) \cdot U(s)
$$

Where $s$ is the Laplace variable and $G_{s c}$ is the transfer function connecting the amplitude $U$ and the scanning position $x_{s c}$. When the voltage is abruptly removed, the resulting step $x_{\text {step }}$ is smaller than the corresponding amplitude $x_{s c}^{U}$ so that [12](Fig. 3 -a):

$$
x_{\text {step }}=x_{s c}^{U}-\Delta_{b a c k}
$$

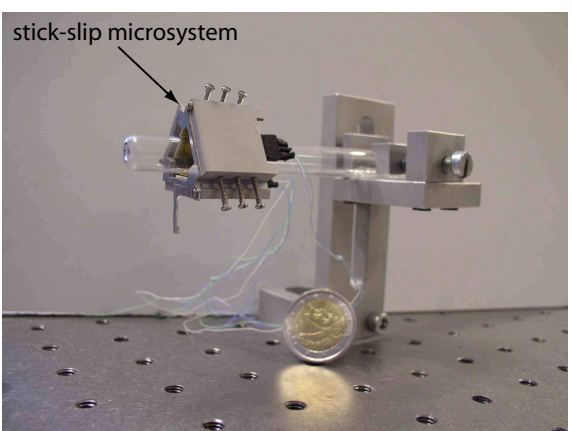

(a)

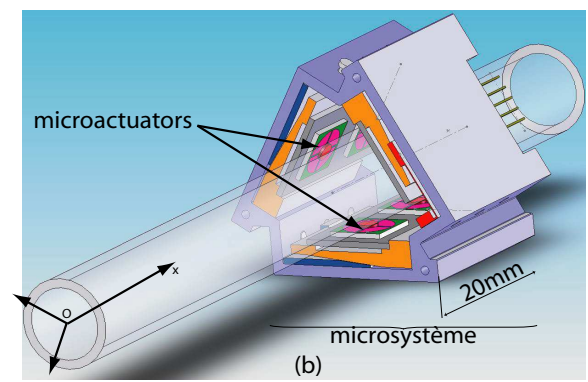

Fig. 2. The stick-slip microsystem.

Hypothesizing that the flashback $\Delta_{b a c k}$ is dynamically linear reative to the amplitude $U$, the step can be written as follow:

$$
X_{\text {step }}(s)=G_{\text {step }}(s) \cdot U(s)
$$

When the sequence is repeated (stepping mode) with a frequency $f=1 / T$, the dynamic of the continous part $G_{\text {step }}$ is not visible and the microsystem works with a quasi-static manner. Thus, the step can be approximated by:

$$
x_{\text {step }}=\alpha \cdot U
$$

where $\alpha>0$ is the static gain of $G_{\text {step }}$.

From the Fig. 3-b and the (Eq. 5), we infer the bilinearity of the speed:

$$
v=\dot{x}=\alpha . U . f
$$

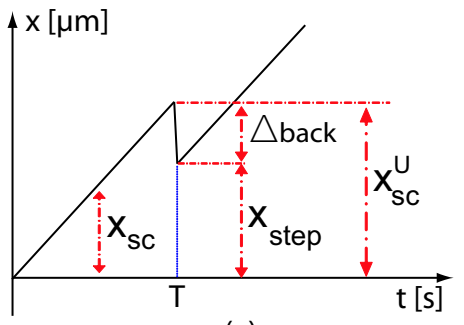

(a)

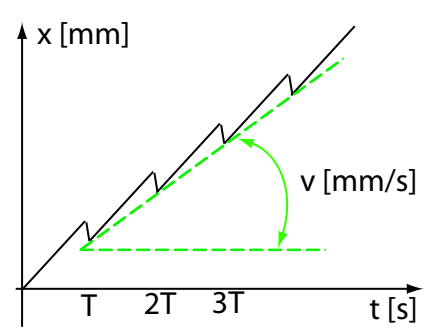

(b)
Fig. 3. a: motion of a stick-slip system. b: speed approximation.

When the amplitude $U$ is below a value $U_{0}$, the torque is not sufficient and the microsystem can't move. Thus, 
an offset is introduced to the (Eq. 5) and the final model becomes:

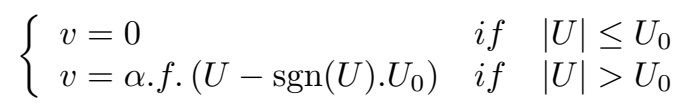

Fig. 4 summarize the speed performances of the microsystem. Until $f=10 \mathrm{kHz}$, the speed is approximately linear versus $f$ (Fig. 4-a). Above this frequency value, a saturation and a fluctuation appear. The identified parameters are: $\alpha=15.652173 \times 10^{-7}$ and $U_{0}=35 \mathrm{~V}$ (Fig. 4-b).
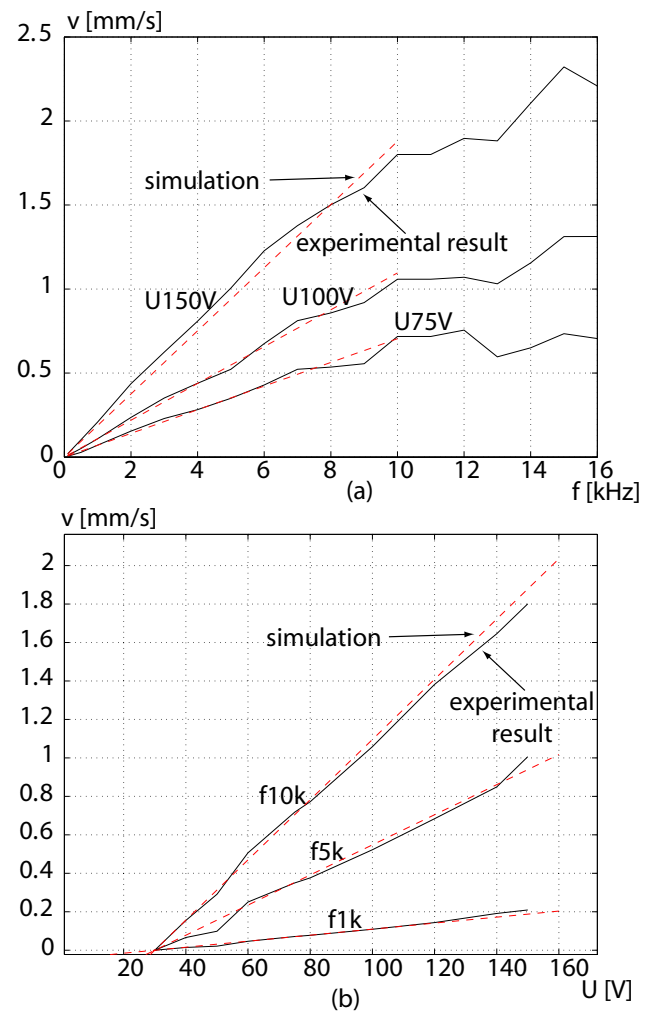

Fig. 4. Speed performances of the microsystem. a: speed versus the frequency $f$. b: speed versus the amplitude $U$.

\section{U/F PROPORTIONAL CONTROL}

\section{A. Principle scheme}

The principle scheme of $\mathrm{U} / \mathrm{f}$ proportional control is shown in Fig. 5. The saturations avoid the overvoltages and limit the microsystem work inside the linear zone of the frequency. The proportional gains $K_{U}>0$ and $K_{f}>0$ are to be adjusted like in a classical proportional controller.

\section{B. Equations and analysis}

In this section, we express the speed according to the values of $K_{U}$ and $K_{f}$. Let $U_{s}$ and $f_{s}$ indicate the saturations respectively used for the voltage and for the frequency. a)

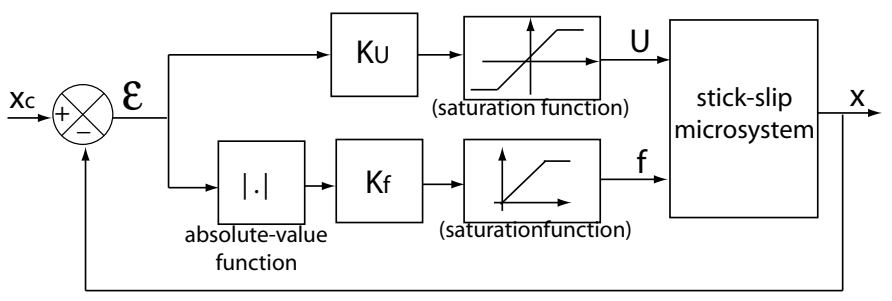

Fig. 5. Principle scheme of the U/f proportional control.

if $K_{U} \cdot\left|x_{c}-x\right|>U_{s} \quad$ and $\quad K_{f} \cdot\left|x_{c}-x\right|>f_{s}$ From the Fig. 5 and the bi-afine expression in (Eq. 7), we obtain:

$$
\dot{x}=\alpha \cdot f_{s} \cdot\left(U_{s}-U_{0}\right) \cdot \operatorname{sgn}\left(x_{c}-x\right)
$$

This case is equivalent to a sign control (Fig. 6-a). When the microsystem is at the neighborhood of the final position, there are oscillations in sign control. The frequency and the amplitude of these oscillations depend on the response time $T_{r}$ of the process, on the refreshing time $T_{s}$ (Fig. 6-b) of the controller and on the frequency saturation $f_{s}$. It is the way that realtime feedback systems give the best performances.

b) if $U_{0}>K_{U} \cdot\left|x_{c}-x\right| \quad \forall f=K_{f} \cdot\left|x_{c}-x\right| \quad$ we have:

$$
\dot{x}=0
$$

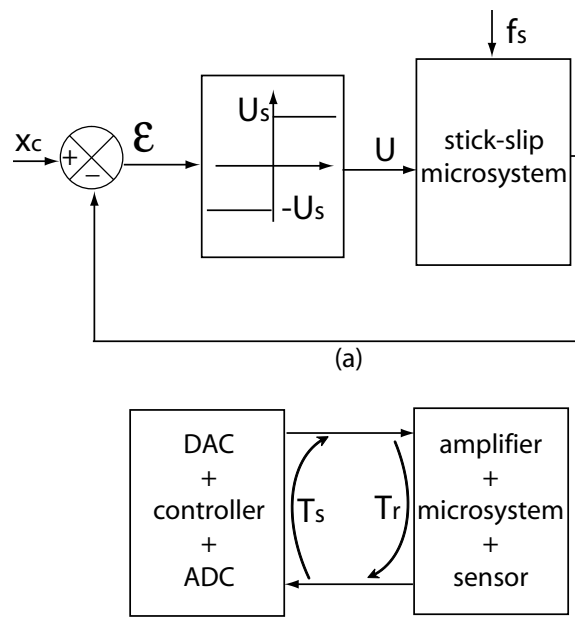

(b)

Fig. 6. a: sign control. b: oscillations in sign control.

c) if $U_{s} \geq K_{U} \cdot\left|x_{c}-x\right| \geq U_{0} \quad$ and $\quad K_{f} \cdot\left|x_{c}-x\right|>f_{s}$ Here, the closed loop process is equivalent to a voltage proportional control (Fig. 7), also known as proportional control in the control theory.

The equation of the closed loop is:

$$
\dot{x}=\alpha \cdot f_{s} \cdot\left(K_{U} \cdot\left(x_{c}-x\right)-\operatorname{sgn}\left(x_{c}-x\right) \cdot U_{0}\right)
$$

For simplification but without loss of generality, let us take a positive target position $x_{c}$ and an initial value 
$x(t=0)$ equal to zero, we obtain the following Laplace transformation:

$$
X=\frac{1}{1+\frac{1}{\alpha \cdot f_{s} \cdot K_{U}} \cdot s} \cdot X_{c}-\frac{\frac{1}{K_{U}}}{1+\frac{1}{\alpha \cdot f_{s} \cdot K_{U}} \cdot s} \cdot U_{0}
$$

where $s$ is the Laplace variable. The (Eq. 11) means that the closed loop process is a first order dynamic system with a static gain equal to unity and a disturbance $U_{0}$. We can infer that, the more $K_{U}$ is great, the more a voltage proportional control of a stick-slip system is accurate. The voltage proportional control always gives stability because it is a first order.

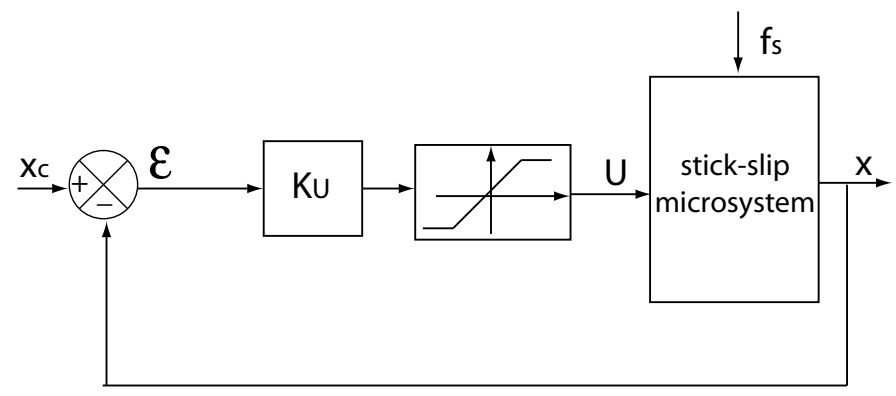

Fig. 7. Voltage proportional control.

d) if $K_{U} \cdot\left|x_{c}-x\right|>U_{s}$ and $f_{s} \geq K_{f} \cdot\left|x_{c}-x\right|$ This case is equivalent to a frequency proportional control (Fig. 8).

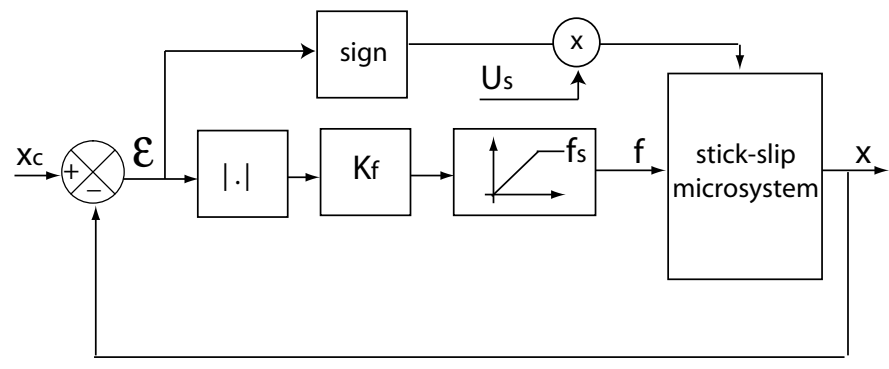

Fig. 8. Frequency proportional control.

The following equation is easily obtained:

$$
\dot{x}=\alpha \cdot K_{f} \cdot\left|x_{c}-x\right| \cdot\left(U_{s}-U_{0}\right) \cdot \operatorname{sgn}\left(x_{c}-x\right)
$$

The expression (Eq. 12) is equivalent to the following transfer function:

$$
\frac{X}{X_{c}}=\frac{1}{1+\frac{1}{\alpha \cdot K_{f} \cdot\left(U_{s}-U_{0}\right)} \cdot s}
$$

The (Eq. 13) differently means that in this case, the closed loop process is a first order system with a static gain equal to unity. The stability is always ensured. e) if $U_{s} \geq K_{U} \cdot\left|x_{c}-x\right| \geq U_{0}$ and $f_{s} \geq K_{f} \cdot\left|x_{c}-x\right|$ The frequency and the voltage are both proportional to the error $\varepsilon$. From the Fig. 5 and the formula (Eq. 7), we have the following expression:

$$
\dot{x}=\alpha \cdot K_{f} \cdot\left|x_{c}-x\right| \cdot\left(K_{U} \cdot\left(x_{c}-x\right)-\operatorname{sgn}\left(x_{c}-x\right) \cdot U_{0}\right)
$$

The expression (Eq. 14) is equivalent to:

$$
\begin{aligned}
& \frac{d x}{d t}=\left(\alpha \cdot K_{f} \cdot U_{0}-\alpha \cdot K_{f} \cdot K_{U} \cdot\left|x_{c}-x\right|\right) \cdot x \\
& +\left(-\alpha \cdot K_{f} \cdot U_{0}+\alpha \cdot K_{f} \cdot K_{U} \cdot\left|x_{c}-x\right|\right) \cdot x_{c}
\end{aligned}
$$

which is in the form of:

$$
\frac{d x}{d t}=A\left(x_{c}, x\right) \cdot x+B\left(x_{c}, x\right) \cdot x_{c}
$$

Where $A$ and $B$ are the state and the input coefficients. Here, the closed loop system has a 1st order pseudo-linear behavior.

\section{Stability}

According to the values of $K_{U}, K_{f}$ and the error $\left(x_{c}-x\right)$, all the above cases may appear during a displacement. Without loss of generality, let us suppose that $x_{c}=0$ and $x(t=0)>0$ for the stability analysis. Let us divide the displacement into two phases (Fig. 9):

- phase-1; the error $\left(x_{c}-x\right)$ is initially high so that the voltage $U$ and the frequency $f$ are both in their saturations (case a)). The speed is constant.

- phase-2; the error becomes smaller and the speed is not yet constant (equivalent to the other cases).

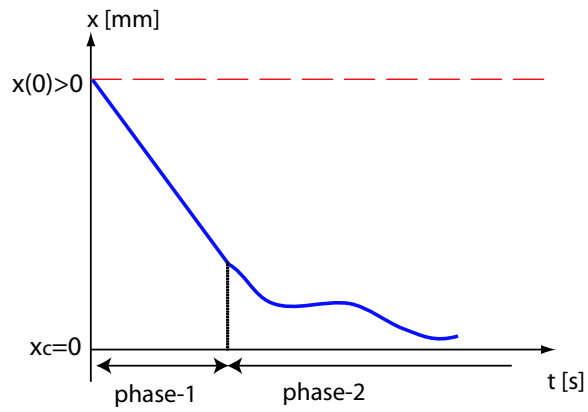

Fig. 9. The displacement may be divided into two phases.

As the equations are quasi-static, ie. there is no acceleration, one case does not influence the succeeding case. So, the phase-2 may be studied independently from the phase-1. In the phase-2, two sub-phases happen:

- phase-2.1: either the frequency is in saturation but not the voltage (case c)) or the voltage is in saturation but not the frequency (case d)),

- phase-2.2: both the frequency and the voltage are not in saturation (case e)).

Once again, the phase-2.1 does not influence the phase2.2. As the possible two cases inside the phase- 2.1 have a 1st order linear behaviors, the displacement from the initial position to current position has not yet an overshoot. On the other hand, when the voltage becomes 
smaller than $U_{0}$ (case b)), the stick-slip microsystem stops independently to the precendent phases. Thus, the stability analysis of the microsystem may be done only with the phase-2.2 (case e)). For that, we use the direct method of Lyapunov.

A dynamic system $\frac{d x}{d t}=f(x, e, t)$, where $e$ is the input vector, is Lyapunov stable if a Lyapunov function $V(x)$ exists such as:

$$
\begin{gathered}
V(x=0)=0 \\
V(x)>0 \quad \forall \quad x \neq 0 \\
\frac{d V(x)}{d t} \leq 0 \quad \forall \quad x \neq 0
\end{gathered}
$$

From the (Eq. 15) and the conditions $x(t=0)>0$ and $x_{c}=0$, we obtain:

$$
\frac{d x}{d t}=-\alpha \cdot K_{f} \cdot x \cdot\left(K_{U} \cdot x-U_{0}\right)
$$

We use the following quadratical functional $V(x)$ :

$$
V(x)=\gamma \cdot x^{2}
$$

where $\gamma$ is a positive constant. The conditions (Eq. 17) and (Eq. 18) are filled.

From (Eq. 20) and (Eq. 21), we have:

$$
\frac{d V(x)}{d t}=-2 \cdot \gamma \cdot \alpha \cdot K_{f} \cdot x^{2} \cdot\left(K_{U} \cdot x-U_{0}\right)
$$

As $\left(K_{U} \cdot x-U_{0}\right)>0$, the condition (Eq. 19) is filled and all the trajectory is asymptotically (Lyapunov) stable. When $\left(K_{U} \cdot x-U_{0}\right)<0$, the microsystem stops, the stability is obvious and the static error is given.

\section{EXPERIMENTS}

The experimental setup is composed of a computer, an amplifier, the microsystem and a laser sensor $(0.5 \mathrm{~nm}$ resolution, $10 \mathrm{~m}$ accuracy). The computer has no real-time operating system (Windows-XP) and we use LabView software for the implementation of the $\mathrm{U} / \mathrm{f}$ controller.

The choice of $K_{U}$ is a compromise. If $K_{U}$ is very low, the static error is high. If $K_{U}$ is very high, oscillations may occur (case a) when the refreshing time $T_{s}$ and the response time $T_{r}$ are not negligible. For all the experiments, the target point $x_{c}=10 \mathrm{~mm}$ and the initial point $x(0)=0 \mathrm{~mm}$.

The first experiment are done with high values of $K_{u}$ and $K_{f}$. They have been chosen so that the phase-2 never happen. It is equivalent to the (case a). The Fig. 10-a gives the experimental and the simulation curves. Due to the fact that the computer has a non real-time operating system, the experimental results have oscillations (Fig. 10- in solid plot).

Then, we use a low $K_{U}$ and a high $K_{f}$. The frequency stays in saturation while the voltage becomes non saturated when $x_{c}-x$ is inferior to a given value $x_{U S}$ (Fig. 10b) such as $x_{U S}=U_{s} / K_{U}$. In that case, the behavior is equivalent to (Eq. 11) of the case c), ie. a voltage proportional control. As shown in the figure, there is a static error. Its value is equal to $\varepsilon_{\text {stat }}=U_{0} / K_{U}$.
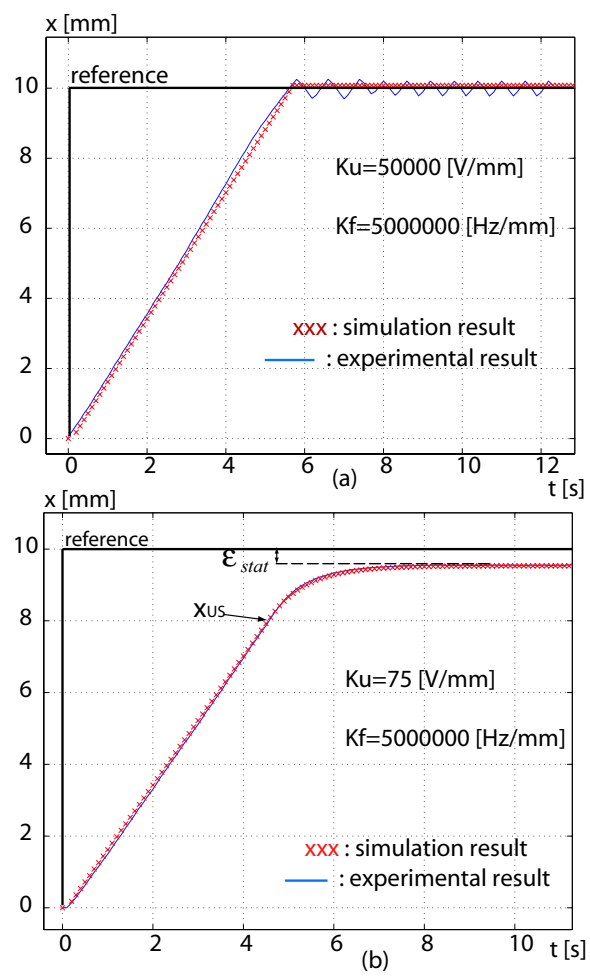

Fig. 10. a: high values of $K_{U}$ and $K_{f}$ : (case a). b: low $K_{U}$ and high $K_{f}$.

After that, we apply a high $K_{U}$ but a low $K_{f}$ (Fig. 11-a). The leaving saturation speed is obtained at $\left(x_{c}-x\right)=x_{f S}=f_{s} / K_{f}$. From this point, the closed loop system has the frequenty proportional controller behavior and there is no static error (case d)).

Finally, we test moderated values of $K_{U}$ and $K_{f}$ (Fig. 11-b). First, the frequency leaves the saturation at $x_{f s}=f_{s} / K_{f}$ while the voltage stays saturated. When $x_{U S}=U_{s} / K_{U}$ is reached, the voltage leaves the satuation. According to the values of $K_{U}$ and $K_{f}$, the inverse circumstance can happen. The static error is given by $\varepsilon_{\text {stat }}=U_{0} / K_{U}$.

\section{Discussion}

The U/f proportional controller encompasses some classical controllers: the sign controller (see case a), the classical proportional controller (see case c) and the frequency proportional controller (see case d). On the other hand, in comparison with the classical algorithm of (Eq. 1) and with the frequantial proportional controller, the U/f proportional controller seems to have a high resolution. The resolution obtained with the two formers is one step (respectively $n \times$ step) while the one obtained with the $\mathrm{U} / \mathrm{f}$ controller is better than one step, within the limits of the sensor accuracy. In fact, the diminution of the applied voltage $U=K_{U}$. $\varepsilon$ reduces the value of a step. In addition, the diminution of the frequency $f=K_{f} . \varepsilon$ reduces the number of the steps and then the vibrations. 

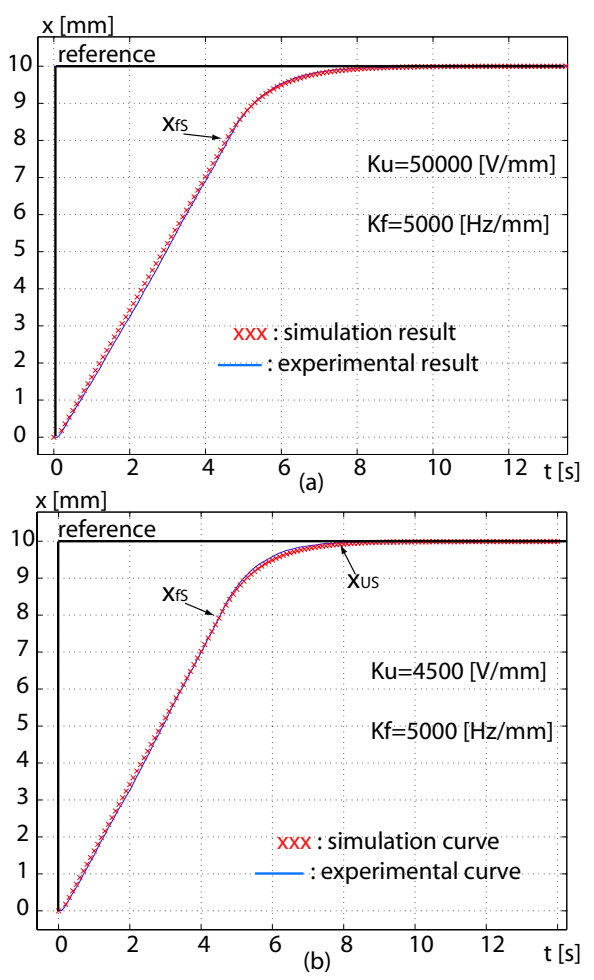

Fig. 11. a: high $K_{U}$ and low $K_{f}$. b: decent values of $K_{U}$ and $K_{f}$.

A test has been performed with an interferometer sensor (resolution $=1.24 \mathrm{~nm})$ to compare the static precision obtained with the algotrithm in (Eq. 1) and the U/f proportional controller. The setup used is composed of materials with high values of $T_{s}$ and $T_{r}$ : the interferometer datagate and a LabView software under WindowsXP. Thus, we use a low frequency $(1 H z)$ for the classical controller in order to avoid oscillations.

Fig. 12 shows the results with a setpoint (reference) of $10 \mathrm{~m}$. The zoom (Fig. 12-b) indicates that the static error (accuracy) is about $130 \mathrm{~nm}$. As we can see, the resolution before the stop is the half value of a step instead of a step because the applied voltage is a peak-to-peak signal $( \pm 150 \mathrm{~V})$, then when stopping it, the half only is cut out $(+150 \mathrm{~V} \rightarrow 0 \mathrm{~V})$.

Fig. 13 gives the results obtained with the U/f controller when applying a setpoint of $1 \mathrm{~mm}$. The zoom (Fig. 13-b) indicates that the frequency and the step amplitude become increasingly small. The medium static error also becomes increasingly small. However, the flashback $\Delta$ back becomes increasingly important. That is due to the diminution of the amplitude $U$ so that there will not be enough torque to move the microsystem during the slip phase. The proposed method appears to converge to the reference position much faster than the classical controller. That is due to the low frequency used for this latter. Increasing the frequency increases the speed of the classical controller but oscillations may appear.
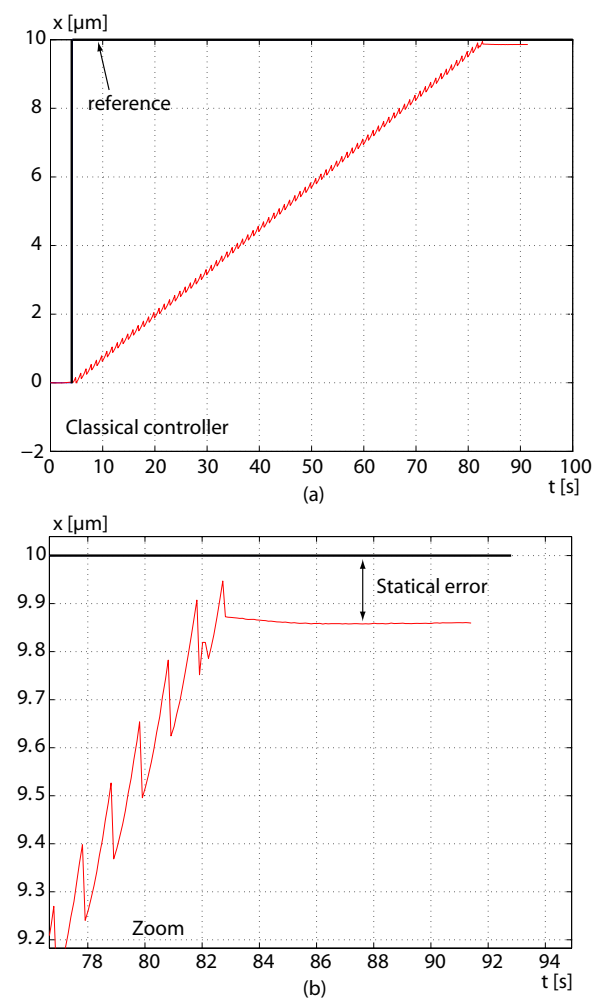

Fig. 12. Classical controller using an interferometer sensor. a: high stroke motion. b: zoom of the final position.

\section{CONCLUSION}

A new controller type for high stroke displacement of stick-slip microsystems was proposed: voltage/frequency proportional controller. Here, the frequency $f$ and an amplitude $U$ of a sawtooth signal are proportional to the error. To analyze the performances given by such controller, we have first modeled the high stroke displacement of the microsystem. Then, we have analysed the stability according to the value of the proportional gains $K_{U}$ and $K_{f}$. Finally, we have presented experiments and simulations of different cases. The $\mathrm{U} / \mathrm{f}$ proportional control is a globalization of existing some existing controllers, the sign controller, the classical proportional controller and the frequency proportional controller, and it gives a better resolution relative to existing controllers for stick-slip microsystems.

\section{ACKNOWLEDGMENT}

Particular thanks to Dr Jean-Marc Breguet and his team from the LSRO - EPFL (Lausanne Switzerland) for accepting us to use their interferometer sensor. This work is partially supported by the European Project EUPASS (http://www.eupass.org/) 

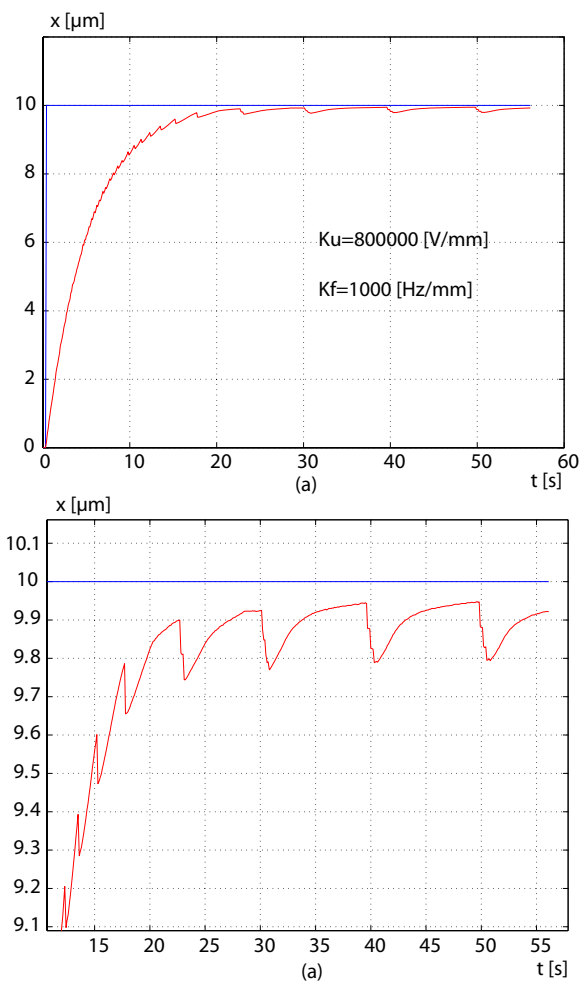

Fig. 13. U/f controller using an interferometer sensor. a: high stroke motion. b: zoom of the final position.

\section{REFERENCES}

[1] J.M Breguet, "Actionneurs 'Stick and Slip' pour micromanipulateurs" PhD Thesis No1756, Ecole Polytechnique Fédérale de Lausanne, Switzerland (in French), 1998.

[2] R. Matsuda and R. Kaneko, "Micro-step XY-stage using piezoelectric tube actuator" IEEE Micro Electro Mechanical Systems Conference Proceedings, pp137-142, 1991.

[3] H. Langen, J.M. Breguet, M. Moscatelli, R. Mericio, Ph. Renaud and H. Bleuler, "Stick-Slip actuators for micromachining of glass" International Conference on Micromechatronics for Information and Precision Equipment, pp261-264, 1997.

[4] A. Bergander,W. Driesen, T. Varidel, M. Meizoso, and J.M. Breguet, "Mobile cm3-microbots with tools for nanoscale imaging and micromanipulation", Mechatronics Robotics, 2004.

[5] S. Fatikow, B. Magnussen, and U. Rembold, "A piezoelectric mobile robot for handling of microobjects", Microsystems, Intelligent Materials and Robots (MIMRS), 2005

[6] A. Bergander, J.M. Breguet, C. Schmitt and R. Clavel, "Micropositioners for microscopy applications based on the stick-slip effect" IEEE International Symposium on Micromechatronics and Human Science, pp213-216, 2000.

[7] A. Bergander and J.M. Breguet, "Performance improvements for stick-slip positioners" Proceedings of 2003 IEEE International Symposium on Micromechatronics and Human Science (MHS), 2003.

[8] J.M. Breguet, R. Pérez, A. Bergander, C. Schmitt, R. Clavel and H. Bleuler, "Piezoactuators for motion control from centimeter to nanometer" Proceeding of International Conference on Intelligent Robots and Systems, IROS, pp-492-497, 2000.

[9] B. Sedghi, "Control design of hybrid systems via dehybridization" PhD Thesis No2859, Ecole Polytechnique Fédérale de Lausanne, Switzerland 2003.

[10] J.M. Breguet and R. Clavel, "Stick and slip actuators: design, control, performances and applications" IEEE Internationam Symposium on Micromechatronics and Human Science, pp-8995, 1998.

[11] M. Rakotondrabe, Y. Haddab and P. Lutz, "Design, devbelopment and experiments of a high stroke-precision 2Dof (linear- angular) microsystem" IEEE ICRA, Orlando Florida, pp-669674, May 2006.

[12] W. Driesen, A. Bergander, T. Varidel and J.M. Breguet, "Energy consumption of piezoelectric actuators for inertial drives" IEEE International Symposium on Micromechatronics and $\mathrm{Hu}-$ man Science, 2003. 